\title{
Development of a spectroscopic diagnostic tool for electric field measurements in IShTAR (Ion cyclotron Sheat Test ARrangement) ${ }^{a)}$
}

\author{
A. Kostic, ${ }^{1,2, b)}$ R. Dux, ${ }^{2}$ K. Crombé, ${ }^{1,3}$ A. Nikiforov, ${ }^{1}$ R. Ochoukov, ${ }^{2}$ I. Shesterikov, ${ }^{2}$ E.H.Martin, ${ }^{4}$ J-M. \\ Noterdaeme, ${ }^{1,2}$ and the IShTAR team ${ }^{2,1}$ \\ 1) Department of Applied Physics, Ghent University, Belgium \\ 2) Max-Planck-Institut für Plasmaphysik, Garching, Germany \\ 3) LPP-ERM-KMS, TEC partner, Brussels, Belgium \\ 4) Oak Ridge National Laboratory, Oak Ridge, Tennessee, USA
}

IShTAR, Ion cyclotron Sheath Test ARrangement, is a linear device dedicated to the investigation of the edge plasma - ICRF (Ion Cyclotron Range of Frequencies) antenna interactions in tokamak edge-like conditions and serves as a platform for a diagnostic development for measuring the electric fields in the vicinity of ICRF antennas. We present here our progress in the development of an optical emission spectroscopy method for measuring the electric fields which concentrates on the changes of the helium spectral line profiles introduced by the external electrical field, i.e. the Stark effect. To be able to fully control the operating parameters, at the first stage of the study the measurements are conducted on a planar electrode installed in the centre of the plasma column in IShTAR's helicon plasma source. At the second stage, the measurements are performed in the vicinity of IShTAR's ICRF antenna.

\section{INTRODUCTION}

Auxiliary heating and current drive with ICRF antennas (Ion Cyclotron Range of Frequencies) is successfully and routinely applied in fusion devices with magnetic confinement. Furthermore, these antennas will be installed in ITER where they should deliver $20 \mathrm{MW}$ of heating power ${ }^{1}$. Nevertheless, operation of ICRF antennas during a tokamak discharge can lead to several parasitic phenomena ${ }^{2-5}$. These effects, namely enhanced impurity sputtering, formation of hot-spots and RF-power dissipation, arise due to interactions between ICRF waves launched from antennas and the edge plasma. To perfect the performance of this essential heating and current drive system, these unwanted effects have to be minimised or eliminated. This is one of the main challenges of contemporary research focused on the ICRF antennas. Two approaches concentrated on solving these issues are ongoing, in parallel: one is to carefully optimise the design and the operational scenarios of antennas ${ }^{6-8}$, while the other is to study in detail the cause of the listed harmful effects and understand the antenna-plasma interaction with the theoretical models of RF-sheaths ${ }^{9-11}$.

Those theoretical models are presently being developed and are constantly improving. They, however, lack an experimental validation which is not easily obtained and/or interpreted from the measurements done in tokamak geometry. Furthermore, these models require direct measurement of parallel (to the background magnetic field)

\footnotetext{
a) Contributed paper published as part of the Proceedings of the 22nd Topical Conference on High-Temperature Plasma Diagnostics, San Diego, California, April, 2018.

b) Author to whom correspondence should be addressed: ana.kostic@ipp.mpg.de
}
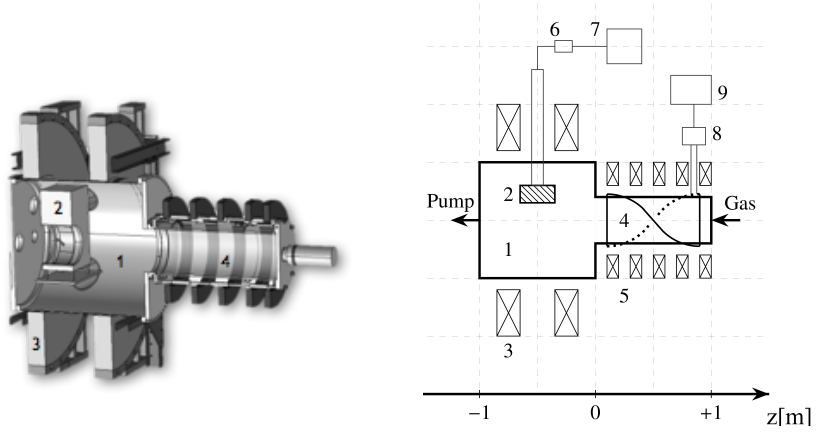

FIG. 1. Realistic (left) and schematic (right) representation of IShTAR: 1 - Main chamber; 2 - single strap ICRF antenna; 3 - Magnetic field coils (Big coils); 4 - Helicon plasma source; 5 - Magnetic field coils (Small coils); 6 - Autotuner; 7 - ICRF generator; 8 - Matching system; 9 - RF generator.

electric field, precisely its DC component, that develops as a result of the so-called $\mathrm{RF}$-sheath rectification, close to antenna structures (limiters and the Faraday screen) on the magnetic field lines that intersect them. It is those DC electric fields that are suspected to be the main cause of enhanced impurity sputtering as they accelerate ions out of plasma into the antenna's boundaries.

To provide required experimental data in simplified geometry, and an easy access for needed diagnostics, a linear device called IShTAR, Ion cyclotron Sheath Test ARrangement, was designed and built at the Max-Planck Institute for Plasma Physics in Garching, Germany. 


\section{A. IShTAR}

IShTAR's main focus is on RF-sheath studies, therefore it is designed to mimic the tokamak edge plasma conditions, and is equipped with a single strap ICRF antenna. It is a linear device with magnetic field produced along the main axis of the machine with the coils in a Helmholtz-like configuration. IShTAR consists of two parts (as depicted in FIG. 1.) - the main vacuum chamber ( $1 \mathrm{~m}$ long and $1 \mathrm{~m}$ in diameter) where the ICRF antenna is located, and the helicon source - a $1 \mathrm{~m}$ long and $0.4 \mathrm{~m}$ in diameter glass tube around which a helicon antenna is wound to enable realisation of plasma density up to $n_{e}=5 \times 10^{17} \mathrm{~m}^{-3}$. The design of the device is elaborated in detail elsewhere ${ }^{12}$.

We will make use of IShTAR's flexibility and the fact that it is designed to match tokamak edge plasma parameters, to develop a diagnostic technique that will enable direct measurements of DC electric fields in RF-sheath in the vicinity of ICRF antennas.

\section{B. Spectroscopic measurements of electric fields}

For this specific task we consider passive optical emission spectroscopy as the diagnostic of choice, mostly because of its non-intrusive nature.

When atoms are exposed to an external electric field their electronic structure is altered, which is known as the Stark effect. This manifests in modified emission spectra - associated spectral lines would shift from the unperturbed wavelength, and the previously forbidden atomic transitions now become allowed which introduces additional "forbidden" spectral lines to appear. These modifications of unperturbed spectra depend on the electric field strength the atoms have been exposed to, and are especially strong for hydrogen and hydrogen-like atoms ${ }^{13}$.

Detection of relative shifts of the spectral lines (with respect to the unperturbed wavelength) of light emitted by excited atoms from the sheath region offer a direct non-intrusive instrument to measure the electric fields these atoms are exposed to.

\section{EXPERIMENTAL METHOD}

\section{A. View-locations and the optical set-up}

In this paper we will discuss two installations within IShTAR. First, to test the feasibility of the method (extension from the feasibility study ${ }^{14}$ ), and re-adjust it for IShTAR plasma and weak electric fields, we have installed a planar stainless-steel disc electrode in the centre of the plasma column in the helicon source. The electrode is then biased with positive voltages up to $3.5 \mathrm{kV}$ in DC regime. The schematics of this set up is given in FIG. 2. Light, from the line of sight indicated in FIG. 2 , is coupled to a $10 \mathrm{~m}$-long $400 \mu \mathrm{m}$ optical fibre through

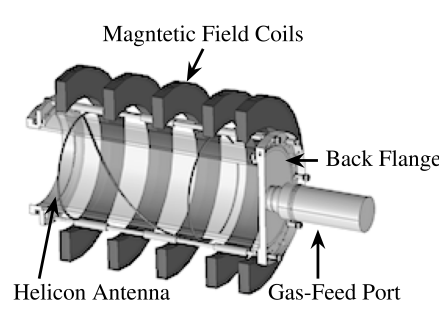

(a) IShTAR's helicon source

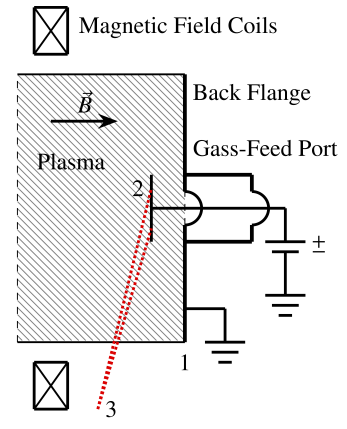

(b) Schematic view of the installed view-location
FIG. 2. Schematics of the electrode set-up installed in the helicon plasma source of IShTAR: 1 - back flange of the machine; 2 - planar electrode; 3 - optical fibre with focused collimating lens and line of sight in dotted line.

an aspherical $6 \mathrm{~mm}$ collimating lens of $8.7 \mathrm{~mm}$ confocal length and an adjustable focal point.

The second set-up was placed in IShTAR's main vacuum chamber. Through a vacuum feedthrough a vacuum-compatible $2 \mathrm{~m}$-long $400 \mu \mathrm{m}$ optical fibre with chrome-plated brass jacketing was installed inside the vacuum chamber, on the holder $2 \mathrm{~cm}$ away from the left side of the ICRF antenna. A collimating lens of the same kind as the one used in the "electrode set-up" described above, was mounted on the optical fibre and focused onto the leading edge of the antenna box (FIG. 3.).

The optical emission spectra from these two viewpoints were recorded with an Andor ${ }^{\text {TM }}$ Shamrock750 spectrometer, of $750 \mathrm{~mm}$ focal length and f/9.7 aperture. The spectrometer is of Czerny-Turner optical arrangement and is equipped with three $68 \times 68 \mathrm{~mm}$ gratings of 600,2400 and 3600 lines/mm, and an intensifying CCD sensor (Andor ${ }^{\mathrm{TM}}$ iStar CCD $334 \mathrm{~T}$ model, with $1024 \times 1024$ active-pixels matrix, and pixel size of $13 \mu \mathrm{m})$. To be able to follow the changes that occur on recorded spectra, we have used a grating with the highest number of lines per mm and centred it on the selected wavelength with a $2.33 \mathrm{~nm}$ bandpass. The analysis of calibration lamp spectra showed that the spectral resolution of this system was $0.0023 \mathrm{~nm}$ per pixel, with the instrumental broadening of $0.01 \mathrm{~nm}$.

\section{B. Selection of the transition}

IShTAR is equipped to use helium and/or argon gases for discharges. For this particular study, the discharges are performed with pure helium due to the following benefits of studying the Stark effect on helium spectral lines:

i. The effect is stronger for atoms where states with different orbital momentum $l$ are degenerate as in hydrogen or nearly degenerate like in helium, thus easier to observe and more sensitive for weaker elec- 


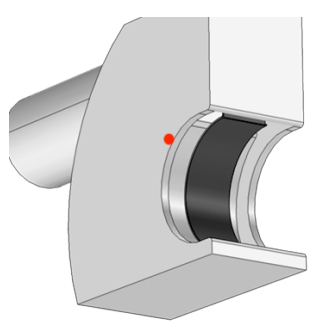

(a) IShTAR's ICRF antenna

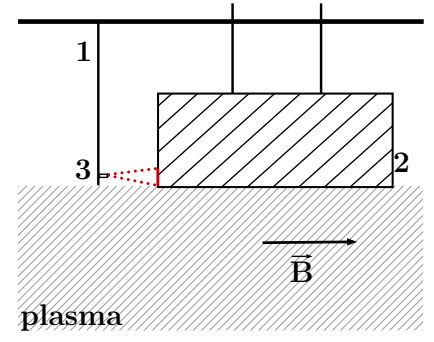

(b) Schematic view of the installed view-location
FIG. 3. View-location on ICRF antenna: The region where the lens is focused on is indicated in (a) in red; on the schematic view of the set-up, (b) the antenna region from FIG. 1 is enlarged to show the fibre and the fibre holder (1) mounted on the wall of the vacuum chamber $2 \mathrm{~cm}$ from ICRF antenna (2) with an indicated lens position (3) and line of sight represented in red.

tric fields.

ii. Both the shift and the forbidden components can occur in helium spectra altered by the Stark effect.

iii. In the electronic configuration of an excited helium atom one electron is always present in the ground state. Since the energy difference between the ground and the first excited state in this configuration is large, the wave-functions of the selected transition can be accurately represented by the wave-function of hydrogen, which simplifies the modelling of the spectral lines with the Schrödinger equation solver.

To select a transition we have to keep in mind that while the sensitivity of an electron to an external electric field is increasing with the prime quantum number of the upper level, at the same time the intensity of the associated spectral line is decreasing. For our experiments, we have selected to focus on the $\mathrm{n}=4 \rightarrow 2$ transition of excited helium atoms, since the signal-to-noise ratio of the recorded spectra was acceptable.

\section{Features of $4^{3} \boldsymbol{D} \rightarrow 2^{3} \boldsymbol{P}$ helium I spectral line profile}

When spectral resolution allows it, recorded spectra of $\mathrm{HeI} 4^{3} \mathrm{D} \rightarrow 2^{3} \mathrm{P}$ line (at $447.1 \mathrm{~nm}$ ) in the absence of external electric field looks like the one shown in FIG. 4. What is indicated as "peak 1" and "peak 2" are in fact 6 transitions convoluted with Gaussian and Lorenzian profiles that arise from the instrumental broadening and contribution from an intensifier in front of the CCD sensor respectively.

To illustrate the Stark effect on this line, a synthetic spectra was calculated using the Explicit Zeeman Stark Spectral Simulator (EZSSS). This code first calculates the perturbed states of the upper and lower level due to the external electric and/or magnetic field and finally

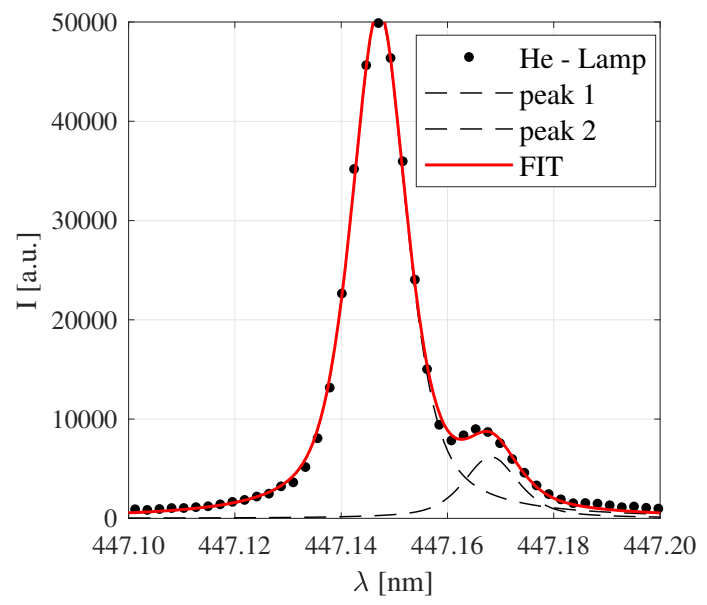

FIG. 4. Spectral line profile of $4^{3} \mathrm{D} \rightarrow 2^{3} \mathrm{P}$ He I transition recorded from the He calibration lamp fitted with pseudoVoigt profile shows characteristic two peaks.

yields a discrete spectrum of all electric dipole transitions. Next, a continuous line profile is obtained by convoluting calculated discrete lines with Gaussian and/or Lorenzian to mimmic the broadening mechanisms that may occur in plasma or are due to instrumental broadening. Detailed description of the atomic theory and mathematical models the code utilises is provided in E.H. Martin's P.h.D thesis manuscript ${ }^{15}$.

Figure 5 shows how the spectral line associated with the He I $4^{3} \mathrm{D} \rightarrow 2^{3} \mathrm{P}$ transition shifts when exposed to electric fields of up to $4 \mathrm{kVcm}^{-1}$ which are expected in our experiments. The forbidden line corresponding to $4^{3} \mathrm{~F} \rightarrow 2^{3} \mathrm{P}$ transition occurs as well at lower wavelengths when electric field is applied, however due to its weak intensity it is not distinguishable form the noise when the real data is collected, and is therefore neglected in this study.

When the same calculation is repeated with a smaller step, the shift of the spectral line with respect to its unperturbed wavelength shows a quadratic increase with increasing electric field strength. In FIG. 6 we have mapped electric field intensity as a function of the shift of the synthetic line profile to obtain the fit function that will later be used to calculate the electric field for the measured shifts of lines recorded during the experiments. Fitting the mean of $\mathrm{d} \lambda$ for both components of the line profile, we have obtained the following dependency:

$$
E(\mathrm{~d} \lambda)\left[\mathrm{kV} \mathrm{cm}^{-1}\right]=62 \times \mathrm{d} \lambda^{0.5}[\mathrm{~nm}]+\mathrm{d} \lambda^{0.25}[\mathrm{~nm}]
$$

where $\mathrm{E}$ is the electric field strength, and $\mathrm{d} \lambda$ - the shift of the line with respect to its unperturbed wavelength.

\section{RESULTS}

The results presented in this section were acquired using time-resolved and line integrated passive optical emis- 


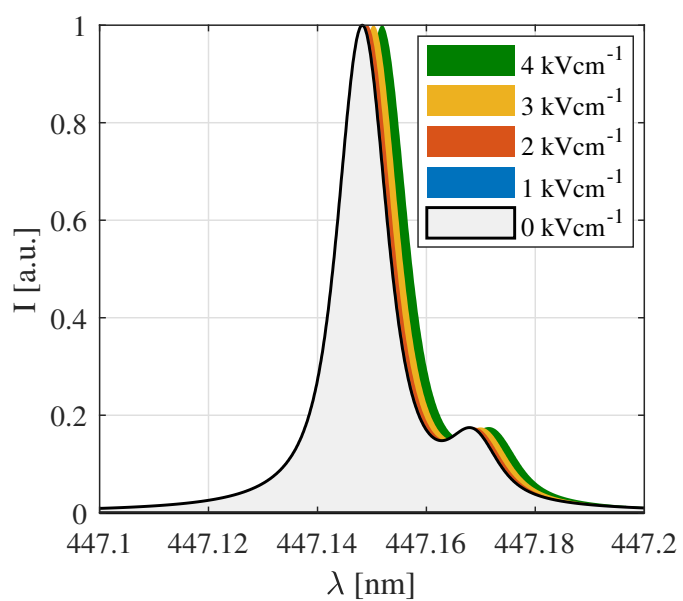

FIG. 5. Synthetic spectra obtained with the EZSSS code shows the characteristic two-peak feature of the spectral line associated with $\mathrm{He} \mathrm{I} 4^{3} \mathrm{D} \rightarrow 2^{3} \mathrm{P}$ transition and the Stark shift with increasing external electric fields used in the calculations.

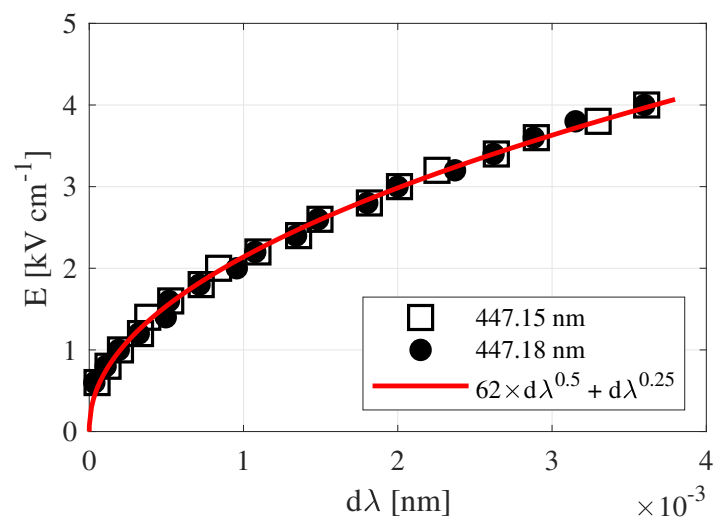

FIG. 6. External electric field intensity as a function of Stark shifts of the two peaks characteristic for the spectral line profile of $\mathrm{He} \mathrm{I} 4^{3} \mathrm{D} \rightarrow 2^{3} \mathrm{P}$ transition with respect to the unperturbed wavelength.

sion spectroscopy along the line of sight from the two previously described view-locations. It has to be noted that the effect of the electric field is local, from the sheath region in front of the electrode or the ICRF antenna, while the integration effects are contributing to, mainly, Doppler broadening.

\section{A. Electrode}

For the experiments during which the emission was recorded from the electrode region, FIG. 2, the plasma was created with an ICRF antenna and the discharge sustained with the voltage applied on the electrode mounted in IShTAR's helicon source. The helicon antenna was not in operation. The magnetic field was also not present, as

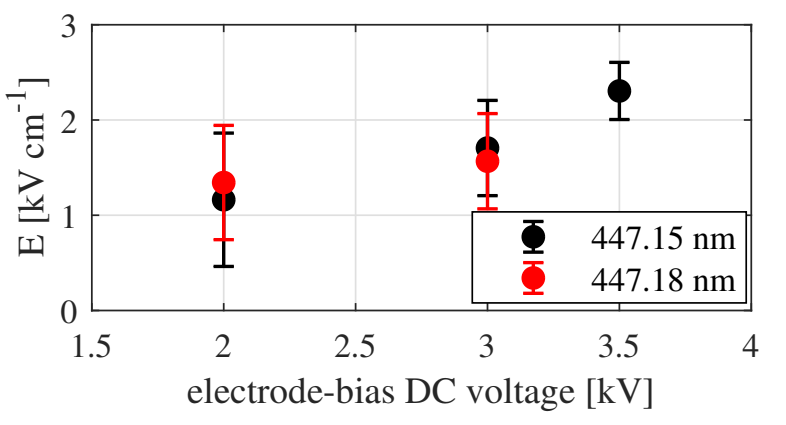

FIG. 7. Electric field calculated based on Eq. 1 for the Stark shifts of spectral lines measured for different voltages applied on the electrode.

its presence significantly lowered the optical signal.

The emission was collected during the entire duration of the discharge with an exposure time of $1 \mathrm{~s}$ per scan, in a kinetic series of 15 scans per discharge. To compare and time-average the recorded data, the scans corresponding to the stable plasma phase were selected and isolated, which was during a reproducible interval of $9 \mathrm{~s}$, yielding 10 scans per discharge. Once the reproducibility of the line was confirmed, we averaged over 5 discharges per scenario. In each scenario we have maintained the same base pressure and flow rate of helium gas, at $\mathrm{p}=10^{-3} \mathrm{mbar}$ and $\mathrm{fr}=60 \mathrm{sccm}$ respectively, while changing the voltage applied on the electrode.

Fitting each time-averaged spectra with a pseudoVoigt profile provided the position of the two peaks. Since these discharges were maintained with voltage applied on the electrode, for the unperturbed spectra we used the one from a spectral lamp used to calibrate the wavelength axes and centre the selected line on the detector matrix. Once those two were subtracted, the obtained $\mathrm{d} \lambda$ was inserted back in Equation (1). Electric fields calculated this way, for each of the scenarios, is presented in FIG. 7. The error-bars in the given calculations come from the uncertanties of the fitting routine and the calibration process and were calculated using the error propagation.

\section{B. ICRF antenna}

When the data was recorded at the ICRF antenna view-location, the plasma was created and maintained in the same manner as in the previously described setup, except here the magnetic field was created with the secondary set of coils (the Small Coils, FIG. 1). The maximal achivevable power, in this plasma configuration, of $1 \mathrm{~kW}$ was delivered to the ICRF antenna that was operated at $5.94 \mathrm{MHz}$ frequency. The base gas pressure was kept constant at $\mathrm{p}=10^{-3}$ mbar.

The exposure time of the optical set-up was $1.5 \mathrm{~s}$, and the data was recorded in kinetic series at the acquisition frequency of $0.66 \mathrm{~Hz}$. Seven spectra, corresponding to the 


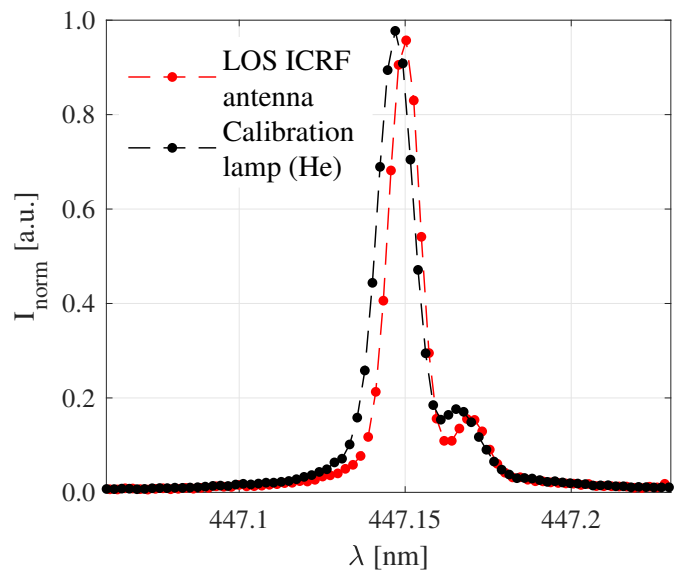

FIG. 8. Ensamble averaged spectra for 7 scans in 19 discharges at the ICRF antenna view-location, normalized for comparison with the He calibration lamp spectra to show the shift from the unperturbed spectra

stable plasma, were isolated per one discharge (FIG.8). At this view-location 19 discharges were recorded. The post-processing of the data was the same as described in the subsection above.

The electric field strength obtaned from the spectral analysis of the data recorded at the leading edge of ICRF antenna powered at $1 \mathrm{~kW}$ was $\mathrm{E}=3.4 \pm 0.3 \mathrm{kV} \mathrm{cm}^{-1}$.

\section{DISCUSSION}

To design a tool that will provide the measurements of DC electric fields in the RF sheaths of ICRF antennas we are using the environment of the IShTAR device, that is built to mimic tokamak edge plasmas, in a linear geometry. Our diagnostic of choice is spectroscopy since it provides direct measurements of electric field without perturbing the conditions in the volume of interest. We have shown in this paper how the Stark effect on the lines of excited helium can be used to fulfil the task, even for fields much weaker than $10 \mathrm{kV} \mathrm{cm}{ }^{-1}$.

The experimental data was collected from two viewlocations in IShTAR, where one was installed on the DC biased electrode in the helicon plasma source, while the other was placed on the side of the ICRF antenna in the main vacuum chamber. We have observed that the relative shifts of the 2 components of the spectral line profile, and thereby electric field, is increasing with increasing voltage delivered to the electrode. With the maximum power delivered to the ICRF antenna, which was of the order of $1 \mathrm{~kW}$, the relative shift of the spectral line profile was larger than observed on the electrode, and the corresponding electric field was found to be $\mathrm{E}=3.4 \pm 0.3 \mathrm{kV} \mathrm{cm}{ }^{-1}$. This behaviour in both presented cases is as expected. Full parameter scans on this view-location are planned.

Nevertheless a few steps are to be taken to minimise the errors - we are constructing at the moment a set-up that will allow measurements of the electric field intensity distribution in the sheath, as it depends on the distance from the electrode. Next, to improve the spectroscopic signal at the ICRF antenna view-location, a local gaspuff is to be installed. This will also allow for helium to be used only for diagnostic purposes, while IShTAR operates with argon plasma which performs better in reaching higher densities needed for achieving the main goal of the machine. Finally, we are working on improving the spectral, temporal and spatial resolution of the measurements in the near future with active Doppler-free Saturation Spectroscopy set-up. The spectral analysis procedure discussed in this paper will, nevertheless, be the same whether we use passive or active spectroscopy to collect the data.

\section{ACKNOWLEDGMENTS}

The authors are grateful to the following for their help with the regular IShTAR operations and this project: F. Fischer, H. Faugel, H. Fünfgelder and G. Siegl.

This work has been carried out within the framework of the EUROfusion Consortium and has received funding from the Euratom research and training programme 2014-2018 under grant agreement No 633053. The views and opinions expressed herein do not necessarily reflect those of the European Commission.

${ }^{1}$ D. W. Swain and R. H. Goulding, Fusion Engineering and Design 82, 603 (2007).

${ }^{2}$ M. Bures, J. Jacquinot, K. Lawson, M. Stamp, H. P. Summers, D. A. D'Ippolito, and J. R. Myra, Plasma Physics and Controlled Fusion 33, 937 (1991).

${ }^{3}$ L. Colas et al., Nuclear Fusion 46, S500 (2006).

${ }^{4}$ S. J. Wukitch, B. Lipschultz, E. Marmar, Y. Lin, A. Parisot, M. Reinke, J. Rice, and J. Terry, Journal of Nuclear Materials 363-365, 491 (2007).

${ }^{5}$ V. Bobkov et al., Nuclear Fusion 50 (2010), 10.1088/0029$5515 / 50 / 3 / 035004$.

${ }^{6}$ V. Bobkov et al., Nuclear Fusion 53, 093018 (2013).

${ }^{7}$ V. Bobkov et al., Plasma Physics and Controlled Fusion 59, 014022 (2017).

${ }^{8}$ W. Zhang et al., Nuclear Fusion 57, 116048 (2017).

${ }^{9}$ J. R. Myra, D. A. D'Ippolito, D. A. Russell, L. A. Berry, E. F. Jaeger, and M. D. Carter, AIP Conference Proceedings 787, 3 (2005).

${ }^{10}$ D. A. D'Ippolito and J. R. Myra, AIP Conference Proceedings 1689, 1 (2015).

${ }^{11}$ D. Van Eester and K. Crombé, Physics of Plasmas 22, 122505 (2015).

${ }^{12} \mathrm{~K}$. Crombé et al., in AIP Conference Proceedings, Vol. 1689 (2015) p. 030006.

${ }^{13}$ H. R. Griem, Principles of Plasma Spectroscopy, 10 (Cambridge University Press, Cambridge, 1997) p. 386.

${ }^{14}$ A. Kostic, K. Crombé, R. D'Inca, J. Jacquot, R. Ochoukov, A. Nikiforov, M. Usoltceva, E. H. Martin, and J.-M. Noterdaeme, EPJ Web Conf. 157, 03025 (2017).

${ }^{15}$ E. H. Martin, Electric field measurements of the capacitively coupled magnetized RF sheath utilizing passive optical emission spectroscopy, Doctor of philosophy, North Carolina State University (2014). 